\title{
Method to Capture and Analyze the Waist-Hip-Thigh Body Region of Seated-Standing 3D Scans
}

\author{
Linsey A. GRIFFIN, Bethany JUHNKE, Emily SEIFERT, Colleen POKORNY, Karolina DORAN \\ University of Minnesota, St. Paul, MN, USA
}

https://doi.org/10.15221/19.254

\begin{abstract}
The purpose of this research was to explore new methods of 3D scanning, body postures, and landmarking techniques to complete in-depth analyses of skin deformation, measurement change, and shape change of the waist-hip-thigh region of the body. There is a need to develop and test new integrated measurement analyses using 1D, 2D, and 3D data to quantify how and where the body is changing in different postures. An integrated approach was taken to select the appropriate 3D scanning technology, develop a landmarking method, and position the body to analyze the waist-hip-thigh region. A convenience sample of 11 women participated in the pilot study, ranging in age from 41-73. Using a quadrant landmarking technique, the body was divided into sections to locally analyze $1 \mathrm{D}$ and $2 \mathrm{D}$ measurements, while conducting volume and curve analysis to aid our understanding of shape change. Local percent change of each circumference was significant, and the data across the various measurements captured the expansion and shrinking of the body. Additionally, the 1D, 2D, and 3D analysis of the models shows the body deforming differently based on participant size, indicating this type of data could be critical for improved size system creation. The results from the extraction of curves represents exciting frontiers in 3D shape research and in the future will enable shape to be more easily incorporated into wearable garments. This data can improve the development of materials, trims, pattern design, and sizing systems. New 3D scanning methods to quantify diverse bodies can improve a company's competitive advantage through enhanced product fit and inclusive, quality design for all.
\end{abstract}

Keywords: 3d body scanning, dynamic anthropometry, functional anthropometry, landmarking

\section{Introduction and Background}

Many anthropometric studies have used 3D scanning technology to capture the human form in a standard A-frame position and seated position [1,2]. However, the in-depth analysis of the full waisthip-thigh region of the body in dynamic poses, such as a seated position, has been limited due to poor scan resolution, lack of color, minimal landmarking, and obstructed views in critical areas such as the hip, inner thigh, and crotch $[3,4,5,6]$. Products and sizing systems must be informed by both the dynamic body and anthropometric data, but traditional anthropometric databases often do not include adequate information about the body in functional postures to make informed engineering and design decisions for close-to-the-body garments in the waist-hip-thigh region. A need exists to improve measurement and shape change data for the waist-hip-thigh area of the body, which is critical to design functional wearable products.

\subsection{Dynamic Anthropometry, Body Measurements, \& Shape Change}

1D and 2D measurements, such as a 34" waist, fail to communicate the body's shape, yet the vast majority of wearable products use traditional anthropometric measurements. This traditional measurement approach to understanding how and where dimension change is occurring on the body is inadequate. Recent research comparing the body in different positions using 3D technology has improved our understanding of how linear measurements change, but the analysis continues to rely on traditional 1D anthropometric methods [1, 2, 7, 8]. When using traditional methods to measure circumferences, despite significant visual shape change of the body, the overall circumference change between positions has been shown to be minimal $[1,9]$.

A disconnect exists between a visual analysis of body shape change and results from traditional 1D anthropometric methods of analysis. A body moves through the expansion of one muscle/s and the contraction on another/s. When examining measurement change of a circumference between two body positions, the measurement fails to capture the body's basic expansion and contraction within one measurement; much of the expansion cancels out the contraction of the body within a circumference measurement. Because of the complexity of how measurements change and shape transform around the body, there is a need to develop and test new integrated measurement analyses using 1D, 2D, and $3 \mathrm{D}$ data to quantify how and where the body is changing in different postures. 


\subsection{Landmarking \& 3D Scanning}

To expand our knowledge of how body measurements change, landmarking methods must first be examined. Traditional landmark locations from the waist to the knee include: waist at front, back, left and right, the tenth rib, iliospinale anterius, max prominence, crotch level, maximum thigh, mid thigh and suprapatellar [10]. Many landmark methods have been developed to capture the body in a standard A-frame and dynamic postures, including the use 3D markers [1], stickers and markers [2, 7], stamps [11], and light projection [12]. Incremental landmark spacing [2, 8] and body crease lines [7] have also been used to track movement of the body. Limitations of these studies include scan positions, obstructed views, and/or no color mapping, which make it difficult to accurately track skin deformation. Measurement techniques for circumferences and shape changes of the hip region and crotch have been limited in scope. Furthermore, 3D scanning research for the body has traditionally employed stationary, full body scanners because of their ability to capture scans of the full body in 10 seconds or less. While the speed is preferred for most studies, the stationary nature of the scanning device typically causes obstructed views of critical areas of the body, especially in the seated position, as illustrated in Figure 1.
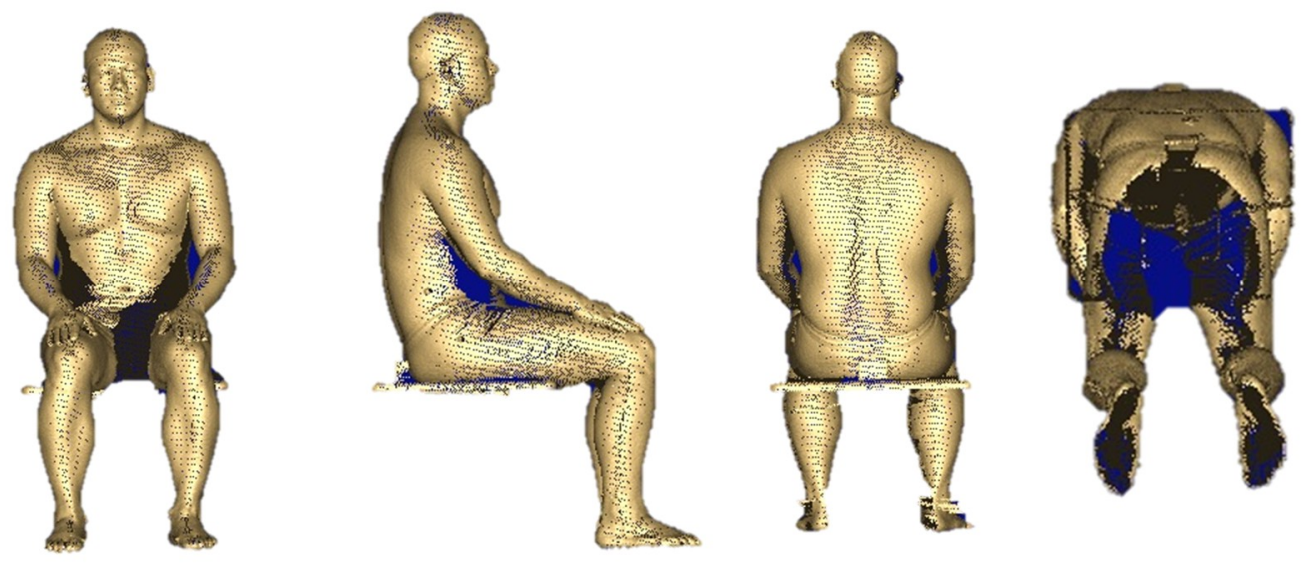

Fig. 1. Images of a seated subject from CAESAR database.

Obstructed views are noticeable in the crotch, hip, and buttocks region. [3]

\subsection{D Scanning Technology}

New handheld 3D scanners use structured light technology to capture a 3D surface texture and color. The handheld scanners make it feasible to collect data in hard-to-see areas of the body such as the crotch and hip. The scanners capture the human body at a faster speed than previous handheld technology, making the analysis of dynamic positions possible. The human body moves and stretches throughout the day to accommodate our busy lives. Analyzing dynamic shape change of the body and product performance requires integrating the scanning technology with new landmarking methods to fully evaluate the 3D complexity of the human body through $1 \mathrm{D}, 2 \mathrm{D}$ and $3 \mathrm{D}$ measurements.

\subsection{Purpose}

The purpose of this research was to explore new methods of 3D scanning, body postures, and landmarking techniques to complete in-depth analyses of skin deformation, measurement change, and shape change of the waist-hip-thigh region of the body. Sectioning a circumference measurement and comparing the percentage change of each section for different postures can provide data-based design decisions for products to act as a second skin. The quality of a garment's design can be improved by first evaluating body shape change. This research seeks to capitalize on the data collected from new 3D scanning technology for anthropometric studies and extend our knowledge of how the body changes in different postures.

\section{Method Development}

\subsection{Overview}

An integrated approach was taken to select the appropriate 3D scanning technology, develop a landmarking method and position the body to analyze the waist-hip-thigh region. Each part of the method was developed to enable an in-depth analysis of circumference measurements and shape change, including the expansion and contraction of body shape across different postures. The overall time of the method was considered for the purposes of a future large population study. 


\subsection{Technology}

The VITUS Smart Full Body Scanner, Artec Eva Scanner, and Occipital Structure Scanner were compared based on the feasibility to capture the human body silhouette through a standing to seated range of motion. The position of the participant, speed to capture a scan, and the technique to landmark the body were evaluated for each scanner.

The waist-hip-thigh region is a complex region of the body to capture dynamic poses with a 3D scanner. Participants must hold a pose for the duration of the scan, with all surfaces of the body visible to the scanner. The VITUS Smart Full Body Scanner had the fastest capture time of 13 seconds per scan compared to the Artec Eva Scanner (30-60 seconds) and Occipital Structure scanner (60-90 seconds) (Table 1). However, the VITUS Smart Full Body Scanner failed to capture surfaces of the body when a participant was not in a standard A-frame position. In a seated position the scan had many incomplete surfaces due to occlusion and was unable to capture the flat surface of the seat region, as the surface is perpendicular to the lasers on the scanner.

The Artec Eva Scanner and Occipital Structure Scanner are mobile scanners that capture the color and texture of a scanned object. The maneuverability of these scanners allows the operator to capture regions and surfaces that are hidden from view with the VITUS Smart Full Body Scanner. Capturing the color and texture on the scanned objects increases the options when landmarking the body. The scan accuracy of these two scanners were compared based on the scan volume to capture the body of a participant. The accuracy of the Occipital Structure Scanner is based on scan volume being captured. The human body requires a larger scan volume for the Occipital Structure Scanner reducing the accuracy of scan data captured, especially when landmarks are placed on the body (Figure 2 center). The Artec Eva Scanner captured various landmarks placed on the body and the scan quality was comparable to a photograph captured of the landmarks. Of the three scanners evaluated, the Artec Eva Scanner captured the best scans for the purposes of analyzing dynamic anthropometric measures of the waist-hip-thigh region of the body.

Table 1. Comparison of specifications for scanners tested to capture dynamic anthropometric measurements.

\begin{tabular}{|c|c|c|c|}
\hline & $\begin{array}{c}\text { VITUS Smart } \\
\text { Full Body Scanner }\end{array}$ & Artec Eva Scanner & Occipital Structure Scanner \\
\hline Scan Time & 13 seconds & 30 to 60 seconds & 60 to 90 seconds \\
\hline Scan Volume & $\begin{array}{c}2.1 \mathrm{~m}(\mathrm{~h}) \times 1.0 \mathrm{~m}(\mathrm{w}) \times 1.2 \mathrm{~m}(\mathrm{~d}) \\
2.772 \mathrm{~m}^{3}\end{array}$ & $61,000 \mathrm{~cm}^{3}$ & Not listed \\
\hline Working Distance & $\sim 0.75 \mathrm{~m}$ & $0.4 m-1 m$ & $0.4 m-3.5 m$ \\
\hline Resolution & -- & $0.5 \mathrm{~mm}$ & $\begin{array}{l}\text { VGA }(640 \times 480) \\
\text { QVGA }(320 \times 240)\end{array}$ \\
\hline Accuracy & $1 \mathrm{~mm}$ & $0.1 \mathrm{~mm}$ & $\begin{array}{l}0.5 \mathrm{~mm} \text { at } 40 \mathrm{~cm} \\
30 \mathrm{~mm} \text { at } 3 \mathrm{~m}\end{array}$ \\
\hline Frame rate & Not available & $1-30 \mathrm{fps}$ & $30 / 60 \mathrm{fps}$ \\
\hline Mobile & No & Yes & Yes \\
\hline Color/Texture & No & Yes & Yes \\
\hline
\end{tabular}
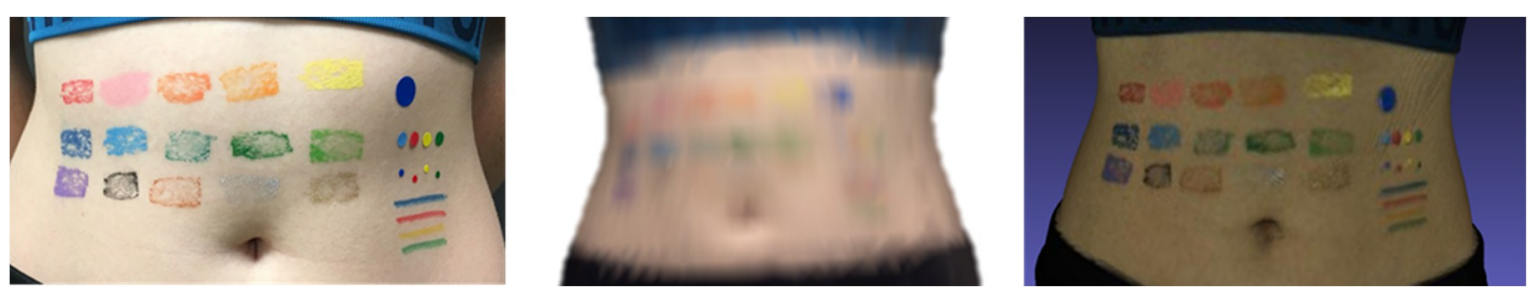

Fig. 2. Photograph of landmarking options (left), scan captured from Structure scanner (center), and scan captured from Artec Eva scanner (right).

\subsection{Body Positions \& Support Apparatus}

\subsubsection{Body Positions}

The movement from standing to seated is one of the most common motions of the waist-hip-thigh region of the body. The seat region of the body is complex to capture with a 3D scanner. The hips fold when seated and, typically, one leg occludes the inner thigh of the other leg. Four positions were analyzed for scanning: traditional a-frame, star, seated, and squat (Figure 3). 


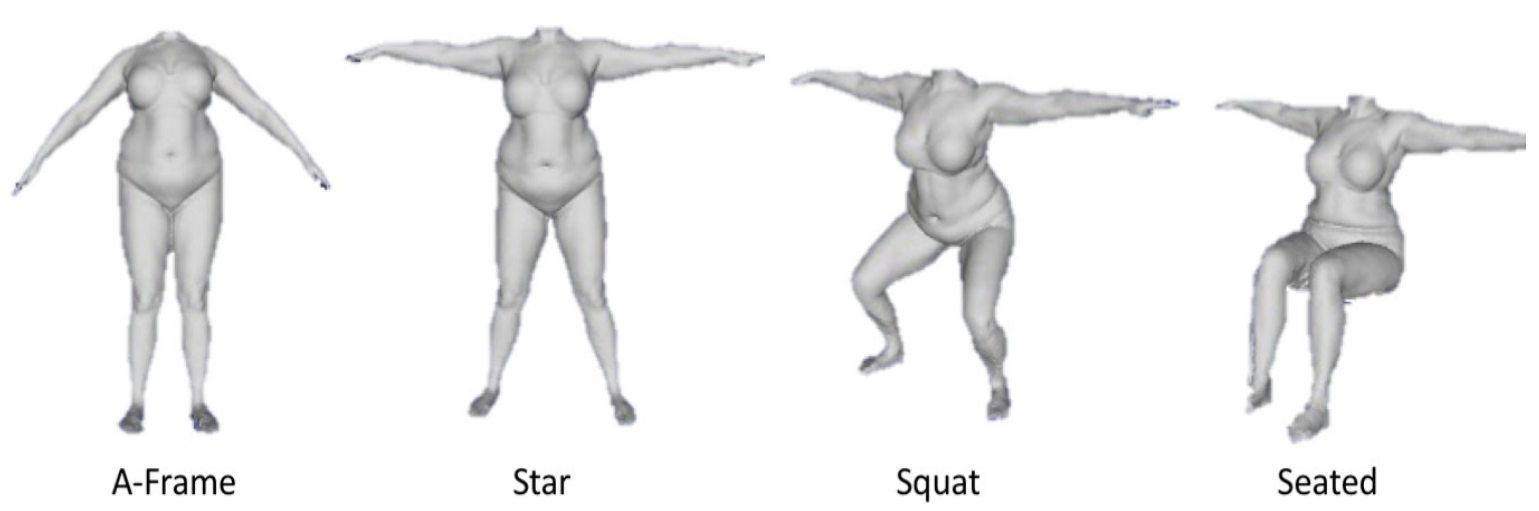

Figure 3. Participant positions considered to study the waist-hip-thigh region of the body.

The a-frame posture positions the participant with their feet hip-width apart and arms at a 45-degree angle from their sides. Automated measurement software is available for a standard a-frame position and the data would be comparable to the existing CAESAR dataset. However, participants' thighs may touch in this position and reduce the quality of measurements during analysis. The star posture positions the participant's feet wider than hip-width at a 20-degree angle measured from the buttock. The scanner has clear views of the participant's inner thighs in the star position to take measurements of the upper thigh, pant rise, and product leg opening, however this non-traditional pose is harder for automated measurement software to analyze.

The squat position starts in the star position and the participant lowers their seat until their thighs are parallel to the floor. A scan of the squat position captures the shape change when moving from standing to seated, however a squat position is hard to hold for the duration of the scan and automated measurement software is not available for this analysis. In the seated posture, the participant looks straight ahead with their feet supported and a 90-degree angle at the knee. Automated measurement techniques are available for a seated position and comparable to CAESAR data, however the seat deforms and obstructs the view of the buttock.

The final positions selected were the star and a seated-star position. The seated-star positions the participant's legs at a 20-degree angle from the sit bones. The scanner captures a better view of the product when the participant is seated with their knees wide.

\subsubsection{Support Apparatus Development}

The stability of participants during the scan is important to capture high quality scans. The Artec Eva Scanner captures hundreds of frames per second to create a model. The movement of a participant mid-scan degrades the quality of the scan, increases the post-processing of the scans, and increases the difficulty to capture measurements. Multiple support apparatuses were designed to stabilize participants during a scan to reduce movement. The selected positions for scanning were the starstanding and the star-seated positions (Figure 4). The decision to reduce the number of positions was based on the amount of time needed to complete the scans and the complexity of supporting one's weight in the squat position.

In the standing position, hiking poles were stabilized with bases for a cane (Figure 5 left). A 50-inch tall plexiglass topped seat was designed to capture scans in a seated position (Figure 5 right). A 0.5 inch plexiglass top was cut to 30 inch $\times 30$ inch to accommodate the maximum seated hip width of 28 -inches. Eight custom cut 29-inch single rail t-slotted frames were assembled for a $30 \times 30$ inch square at the top and bottom of the seat. Four 4-ft long double rails t-slotted frames held up the seat and secured the double rails to the single rails at 16 locations. Either end of the 29 -inch single rail frame sections were supported by one-half of the 4 -ft long double rail legs and secured by two corner surface brackets. Oneinch corner brackets secured the plexiglass to the frame. The seat can support up to a 500-lb participant. Participants use a three-step rolling ladder with a working platform to sit on the scanning seat and footrests are placed to position their knee at a 90-degree angle. The final seat design is easy to maneuver and collapsible for transportation to other study facilities. 

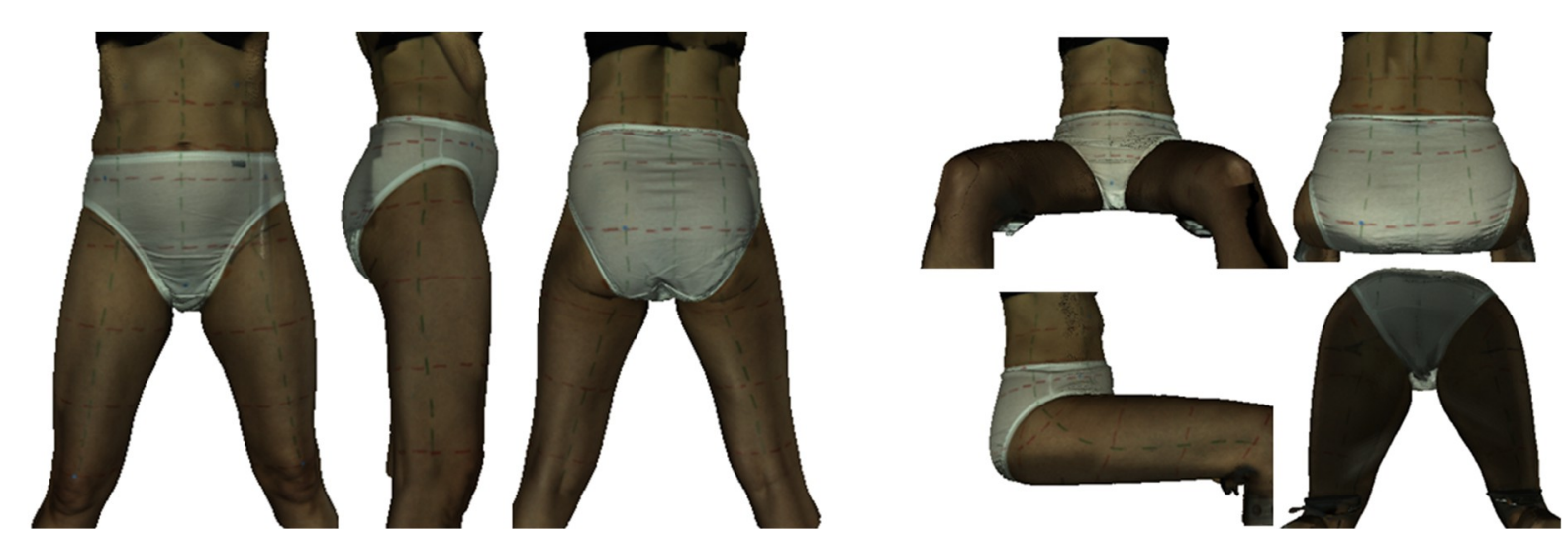

Fig. 4. Example of a scanned participant in standing and seated position with unobstructed views of the waist-hip-thigh region.

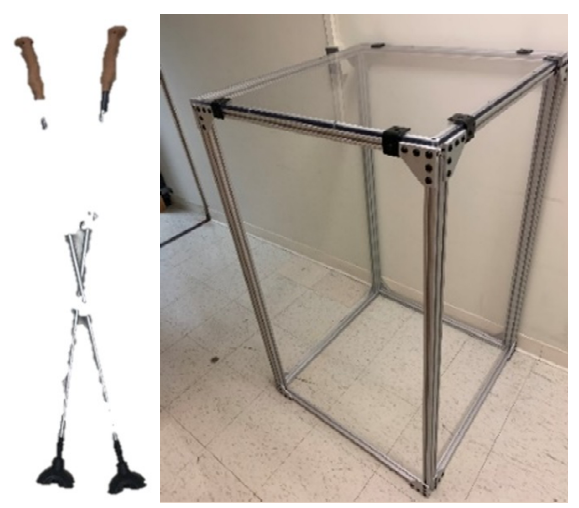

Figure 5. Hiking poles used for stability (left), and supported seat with plexiglass (right).

\subsection{Landmarking}

The waist to knee region is a large section of the body. The chosen landmarking method must be easy to apply to the body, easy to remove from the body, must naturally track to the motion of the body (without restricting or compressing the body), and be valuable for the analysis. The time to complete the landmarking method was critical to the future success of this study. A multi-hour landmarking method is not comfortable for the participant or feasible when enrolling many participants in a study. The landmarking technique must also be minimally invasive due to this sensitive region on the human body. Landmarking garments, which could easily be worn by a participant, may restrict or force the body into unnatural shapes. The purpose of our study is to dynamically analyze the human body to design garments that can be worn close to the body like a second skin, therefore, the data collected must be natural to the human form.

Multiple landmarking techniques from previous studies were analyzed for the waist-hip-thigh region of the body. Five landmark application tools were reviewed; stickers and washable markers [13], 3D landmarking [1], stamps [11], and light projection [12], along with six methods to landmark the body; traditional ISO landmark locations [10], crease and body lines [14, 15], incremental spacing [2], landmarks printed on garments [16], reference grids [17, 8], and stamped speckle patterns [18].

The landmarking evaluation began by applying stickers, markers, and stamps of various colors to the surface of the skin (Figure 2) and capturing the marks with the Artec Eva Scanner. Colors were visually analyzed based on how the color appeared on the skin in the scan and the amount of deformation that was caused by the marking color in the scan. Darker marking colors appeared raised or indented on the surface of the skin in 3D scans; we assumed this deformation was caused by the scanner's algorithm interpreted the color as a shadow or a feature on the skin's surface. Lighter colors were selected for further testing: yellow and green stamp ink, green $1 / 4$ inch diameter circle, and green and red fine line markers.

The traditional ISO landmarking method was the first technique evaluated for the waist-hip-thigh region. Stickers and markers were compared for these landmarks as either dots or lines. Eight landmark locations were identified as important to understand this region of the body: waist (front, back, right, and left), 10th rib, iliospinale anterius, max prominence, max thigh, mid thigh, suprapatella, and crotch crease. The desire to compare these locations from standing to seated inspired using horizontal lines 
at the waist, max prominence, max thigh, mid thigh. The leg crease was marked with a line at the front hip fold and the remaining points were identified with $1 / 4$ inch colored stickers.

An incremental landmarking method was the second technique evaluated. Custom stamps were designed to apply incrementally spaced markings to the body (Figure 6). We found the stamps were difficult to align with one another, align with traditional ISO bony landmarks, and ensure the points were placed symmetrically on the body. The final test scan also appeared to have ghost lines from reading the placement of the stamp in multiple locations, increasing the messiness of the stamps design. Ghost line or messy stamps would decrease the accuracy of the final analysis. We transitioned to incrementally placing circular stamps on the body with larger visibility for the scanner, compared to the stamp designs (Figure 7). Incremental marks on half of the body were applied with the lid to an Elmer's Jumbo Glue Stick and the other half were $1 / 4$ inch circles applied with Crayola Ultra Clean Washable Markers. The marks were placed every 2 inches. The marks showed up well in the test scan but were difficult to place on the body. Tracking the curves of the body added complexity to whether the incremental spacing was aligned horizontally or vertically and how the technique was going to map across different sized bodies within the population. Concern was raised for how the technique could be useful during the analysis, especially when significant variability is present in applying the landmarks to a variety of body shapes. Applying the ink for the stamps was also time consuming and smudged when the participant was in a seated position.

Crease and body lines were the final technique evaluated. The body naturally folds at the front hip crease and lower butt crease when moving from standing to seated. The location of these creases are common areas of complaint for ill-fitting clothing. Product lines were also marked to analyze the product shifting in the seated position. Crease and product lines were marked with a Crayola ultra clean washable marker during the testing.

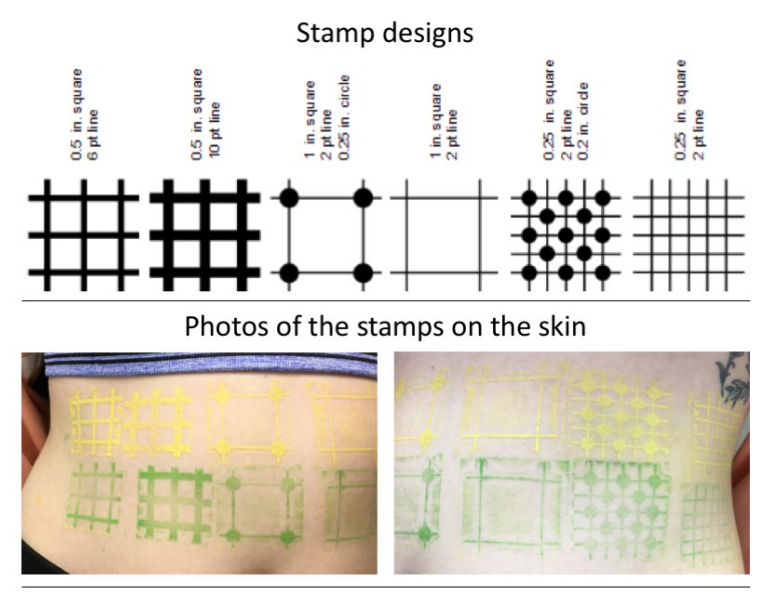

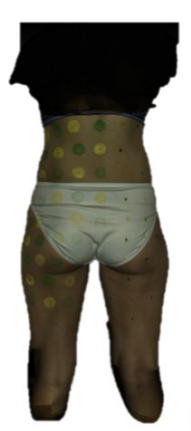

Standing

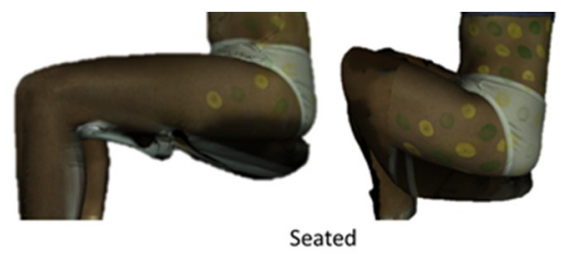

Figure 6. (LEFT) Incremental stamp designs (top), photographs of stamps applied to the body with the yellow and green ink (middle), scan results of stamps applied to the body (bottom). (RIGHT) Incrementally spaced circles placed on the body with ink (left - 1 inch circles) and washable markers (right - $1 / 4$ inch circles).

\subsubsection{Final Landmarking Method}

After testing each of the methods described above, we began merging the methods to optimize the four variables of a landmarking method: color, location (bony or incremental), application technique (sticker, marker, or stamp) and shape (lines or dots). Dynamically evaluating the change in body shape from standing to seated and exploring $2 \mathrm{D}$ and $3 \mathrm{D}$ data about the human body pointed us towards a landmarking method to extract dimension, shape and volume data during the analysis. 

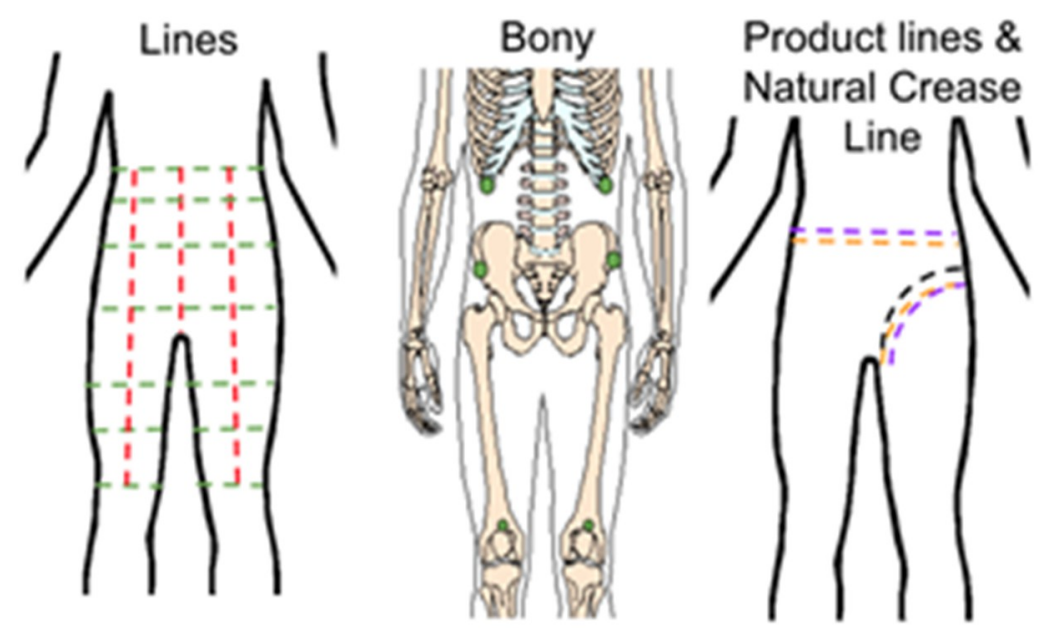

Figure 7. Final landmarking method, which is a combination of 1) horizontal and vertical lines 2) bony landmarks and 3) product and natural crease lines.

The final landmark approach was a combination of drawn horizontal and vertical lines, stickered bony landmarks, and drawn product and natural crease lines (Figure 7). Planes through the body are extracted from horizontal and vertical lines drawn on the body, while the grid shows expansion or contractions at important locations (front and back midline, side seam, waist, hip, thigh, etc.) for product design. Stickered bony landmarks tie the methodology to traditional anthropometric landmarking methods used for research and commonly seen in industry for the design of garments, therefore these scans can function for other research purpose. Product lines show a shift from a standing to a seated position, while natural crease lines must be considered when developing a garment.

\subsubsection{Final Landmarking Method: Lines - horizontal and vertical}

The horizontal and vertical lines on the body were marked with green and red, respectively, using Crayola ultra clean washable markers. Diverse skin tones were tested, and washable metallic markers by Crayola proved to be the most visible application method. A cross line self-leveling laser (Huepar 9011G Cross Line Laser) on a tripod (AmazonBasics Lightweight Camera Mount Tripod Stand) was directed at the participant's body to assist with the line placement. Participants wore safety glasses (Huepar GL01G Green Laser Enhancement Glasses - Eye Protection Safety Glasses) while researchers drew the horizontal and vertical lines on their body. Line placements were selected from anthropometric definitions by SizeUSA [4], ISO 7250-1 [10], or CAESAR [3] datasets and the method of placement was defined for each horizontal or vertical line. The lines were placed on the body in the following order: 1) waist, 2) center front, 3) center back, 4) side seam (left and right), 5) midline front (left and right), 6) midline back (left and right), 7) waist at navel, 8) max hip, 9) high hip, 10) max thigh, 11) knee, and 12) mid-thigh.

\subsubsection{Final Landmarking Method: Crease lines, product lines, bony landmarks}

Next, crease and product lines were drawn on the body. Natural product crease lines of interest were the front leg crease and crease at the bottom of the buttocks. The product lines trace the waistband and leg holes to measure the product shift in the seated position. Finally, stickers are placed to identify bony landmarks: 1) iliospinale anterius (left and right), 2) 10th rib (left and right), 3) max prominence at midlines (left and right), 4) suprapatella, and 5) crotch level. The working definitions of these landmarks was adapted from SizeUSA [4], ISO 7250-1 [10], and CAESAR [3].

\subsection{Population Recruitment}

Participants were recruited through flyers posted throughout all three University of Minnesota Twin Cities campuses, through sponsored advertisements on Facebook, and participants sharing the study with their friends and family. The study inclusion criteria were advertised as women ages 40-65 years of age, although we accepted and were IRB approved for women from 35-75 years of age. As we recruited, we ensured that we had an equal distribution of waist measurements ranging from 28-60". 


\subsection{Integrated Analysis of 3D Scans}

The scans were cleaned and processed with the Artec software. The support apparatuses and scanning noise was removed from the scan data, along with data above the bust or below the knee. Watertight models were created for each scanned position. The models were reviewed to check for double surfaces caused by the slight shift in the skin's surface during scanning or misalignment of the scanning frames in the middle of a scan. The final models were exported as OBJ models with the accompanying MTL and JPG files. On average, it took 30-45 minutes to process the models from one scanning session with a participant. The final scans were zipped and stored on a University managed server, because the size of the files for all participants exceeded the space available on the gaming laptop used to run the Artec software.

\section{Results}

\subsection{Population}

A convenience sample of 11 female Caucasians participated in the pilot for this study. The women ranged in age from 41-73 years of age with an average age of 54 years of age. The participants ranged in height from 1536-1756 mm with an average height of $1659 \mathrm{~mm}$. Their waist measurements ranged from $726-1315 \mathrm{~mm}$, with an average of $1000 \mathrm{~mm}$. Figure 8 shows the range of body shape diversity of the sample.
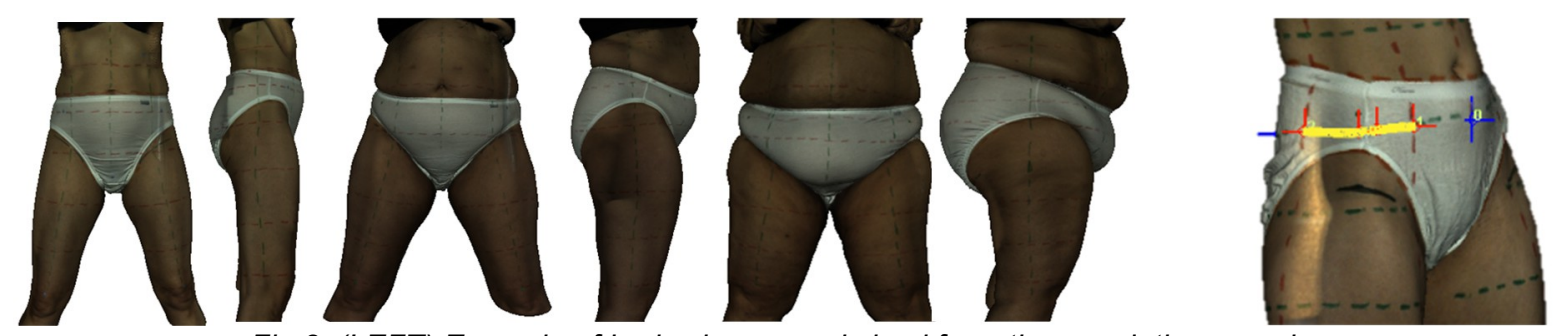

Fig. 8. (LEFT) Example of body shapes and sized from the population sample.

Fig. 9. (RIGHT) Quadrant measurement technique for circumferences.

Measurement shown is the High Hip: Midline Front to Side Seam.

\subsection{Measurement Data}

The 3D scans and landmarking method were used to measure dynamic shape change of the body with respect to a garment's fit. The scans were analyzed visually and quantitatively in Anthroscan, Meshlab, and Materialise 3-matic software, using the landmark quadrants as a reference between the dynamic positions. The quadrants were defined as Center Front (CF) to Midline Front (MF), Midline Front (MF) to Side Seam (SS), Side Seam (SS) to Midline Back (MB), and Midline Back to Center Back (CB). The circumferences were measured as a total circumference, and then each quadrant section was measured in standing and seated position for each scan. A total of 74 measurements were taken per scan using the quadrant measurement technique. The percent change from standing and seated was then calculated for each measurement. Figure 9 shows the results for key circumference measurements and quadrants of the waist, navel, high hip, pant line of underwear, and maximum thigh.

Overall, trends of body expansion and contraction were discovered throughout the dataset, especially at the pant line, thigh, and hip. The expansion and contraction, however, occurred at different rates. The hip circumference increased by $11.32 \%$ overall, and the quadrants show the largest increase occurring from Center Front (CF) to Midline Front (MF) at 18.79\%. The pant line, a measurement that traced the product around the leg opening, showed the most dynamic percent change. Overall the pant line circumference increased by $10.39 \%$, however it decreased by $-18.89 \%$ from the crotch to the midline front (MF), increased from midline front (MF) to side seam (SS) by $9.51 \%$, showed little change $(-.11 \%)$ from the side seam (SS) to midline back (MB), but the measurement increased substantially, $42.97 \%$, from the midline back (MB) to the crotch (Figure 10).

Analyzing local percentage changes in the body can target design decisions for products that act like a second skin. The data across the various measurements indicates that when one area of the body is expanding, another area of the body is shrinking. Additional analysis was conducted on the subjects' measurements based on dividing them up into four size categories. 


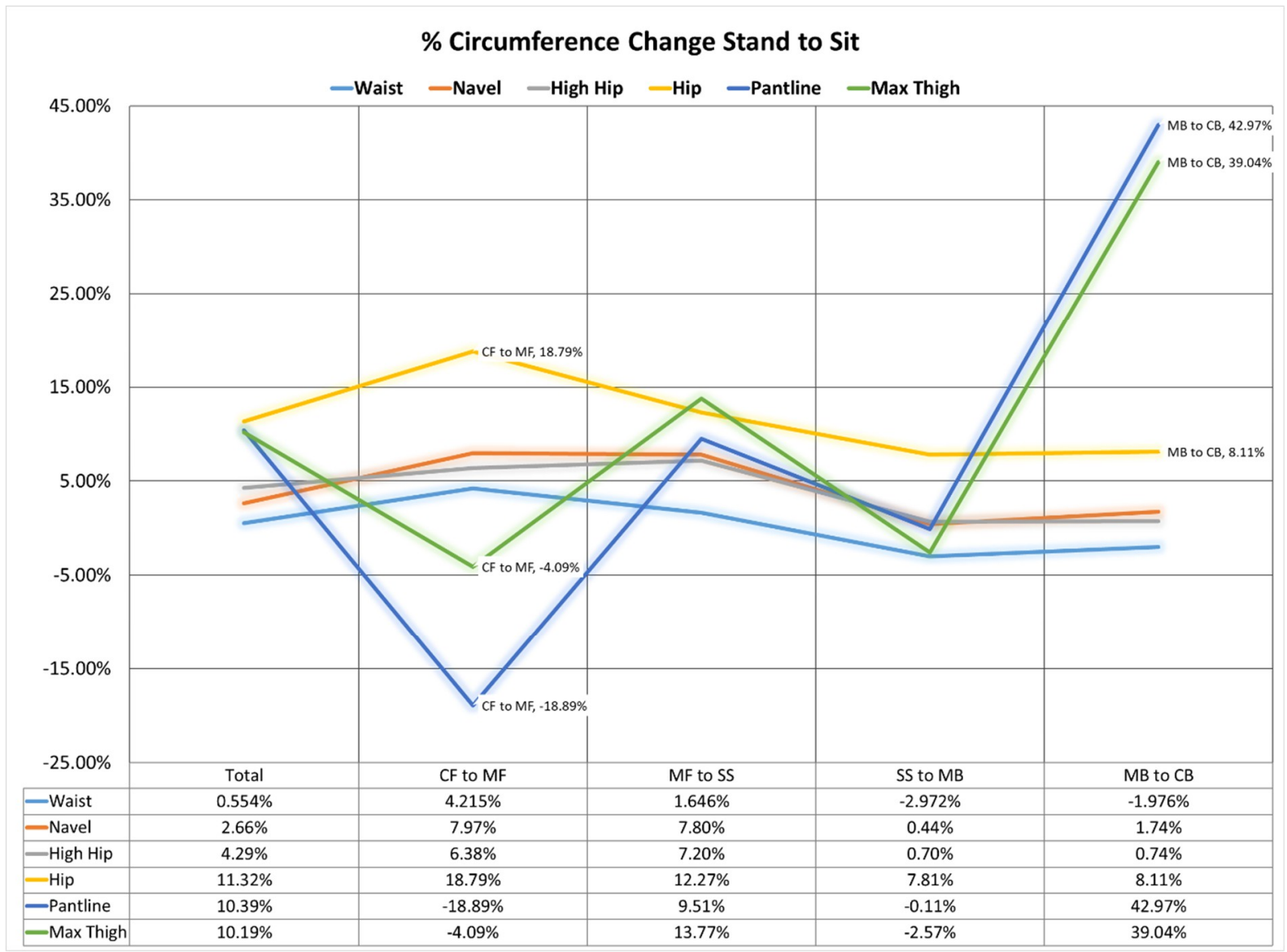

Fig. 10. Circumference change from standing to seated at waist, navel, high hip, hip, pant line, and max thigh.

\subsection{Volume Data}

Each model was cropped at the waist and max thigh to compare how the volume of the body shifts from a standing to a seated position (Figure 11). Additional planes were added at the navel and high hip lines to divide the body into sections. Analyzing the body in sections can improve design decisions for products within each product size.

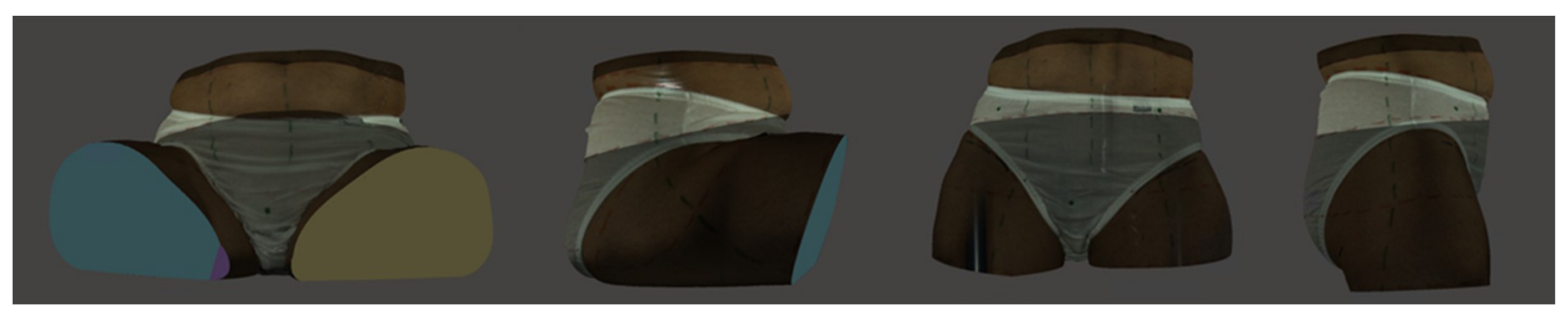

Fig. 11. Standing and seated cropped scan images.

The analysis for the models shows the body deforming differently based on participant size. Breaking the 11 participants into four size categories, trends begin to emerge with the smaller participants showing a decrease in volume from standing to seated, where larger participants showed an increase in volume from standing to seated (Table 2 and Figure 12). The data also showed a correlation between the size of the participant and whether the section increased or decreased in volume. The small and medium participants tended to have a reduction in size towards the top of this section of their body, in which the large and Xlarge participants showed a reduction in volume in the lower section of their body.

Additional sections could be created from the placement of vertical planes. One limitation in our analysis is in the creation of planes in the seated position. In the seated position, the once horizontal lines of the waist, navel, high hip and max thigh have deformed with the body into curved planes. 
Table 2. Volume Percentage Change from Standing to Seated.

\begin{tabular}{|l|c|c|c|c|}
\cline { 2 - 5 } \multicolumn{1}{c|}{} & \multicolumn{4}{c|}{ Volume, Percentage Change from Standing to Seated } \\
\hline Average & Overall Waist to Max Thigh & Waist to Top Line & Top Line to Bottom Line & Bottom Line to Max Thigh \\
\hline Small & $-0.6 \%$ & $-10.6 \%$ & $-0.3 \%$ & $3.7 \%$ \\
\hline Medium & $8.0 \%$ & $13.5 \%$ & $-10.9 \%$ & $10.9 \%$ \\
\hline Large & $5.2 \%$ & $29.8 \%$ & $32.6 \%$ & $-5.7 \%$ \\
\hline XLarge & $5.5 \%$ & $16.5 \%$ & $48.4 \%$ & $-3.3 \%$ \\
\hline
\end{tabular}

*Due to participant anatomical variance, the navel line, and high hip line could be inverted or the same location, therefore the top line or bottom line could be either the navel line or the high hip line.

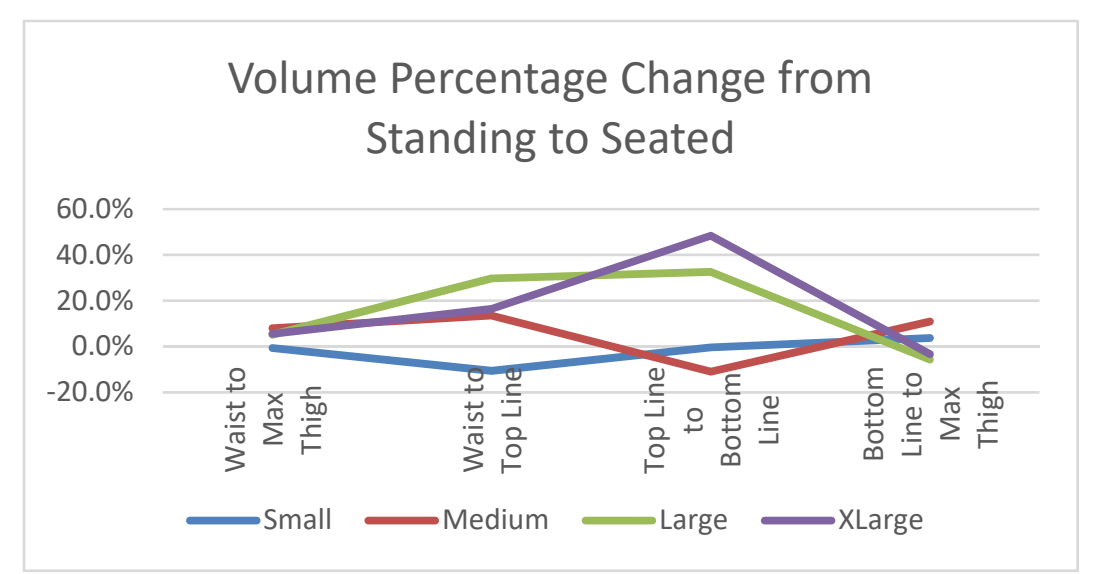

Figure 12. Volume Percentage Change from Standing to Seated.

\subsection{Shape Data}

The curved planes seen during the volume analysis sparked the exploration of extracting curves from the body to reduce visual clutter and begin drawing conclusions about the shape of the human body. Products meant to fit the body like a second skin must be designed to effectively track the deformation of the human body during daily movement. In this analysis, Materialise 3-Matic software was used to extract the waist, hip and pant line curve from participant's scans in a seated and standing position (Figure 13).
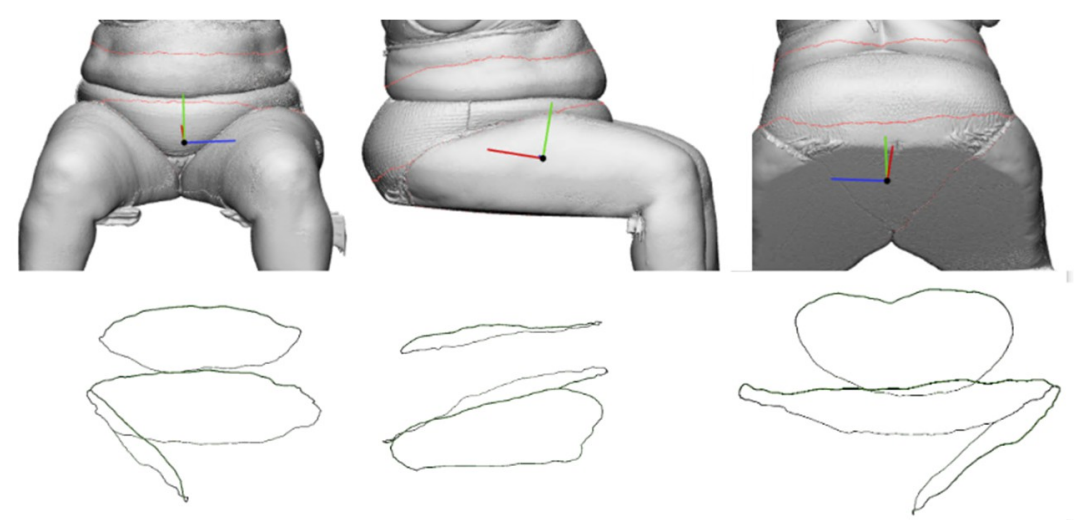

Figure 13. Waist, hip and pant line curves extracted from an XLarge participants seated scan.

The extraction of these curves enables shape to be incorporated into the design of wearable garments (Figure 14). Designs of products worn close to the body can be improved by analyzing the shape profiles of women's bodies across a sizing system and including the analysis into the design of the garment. 


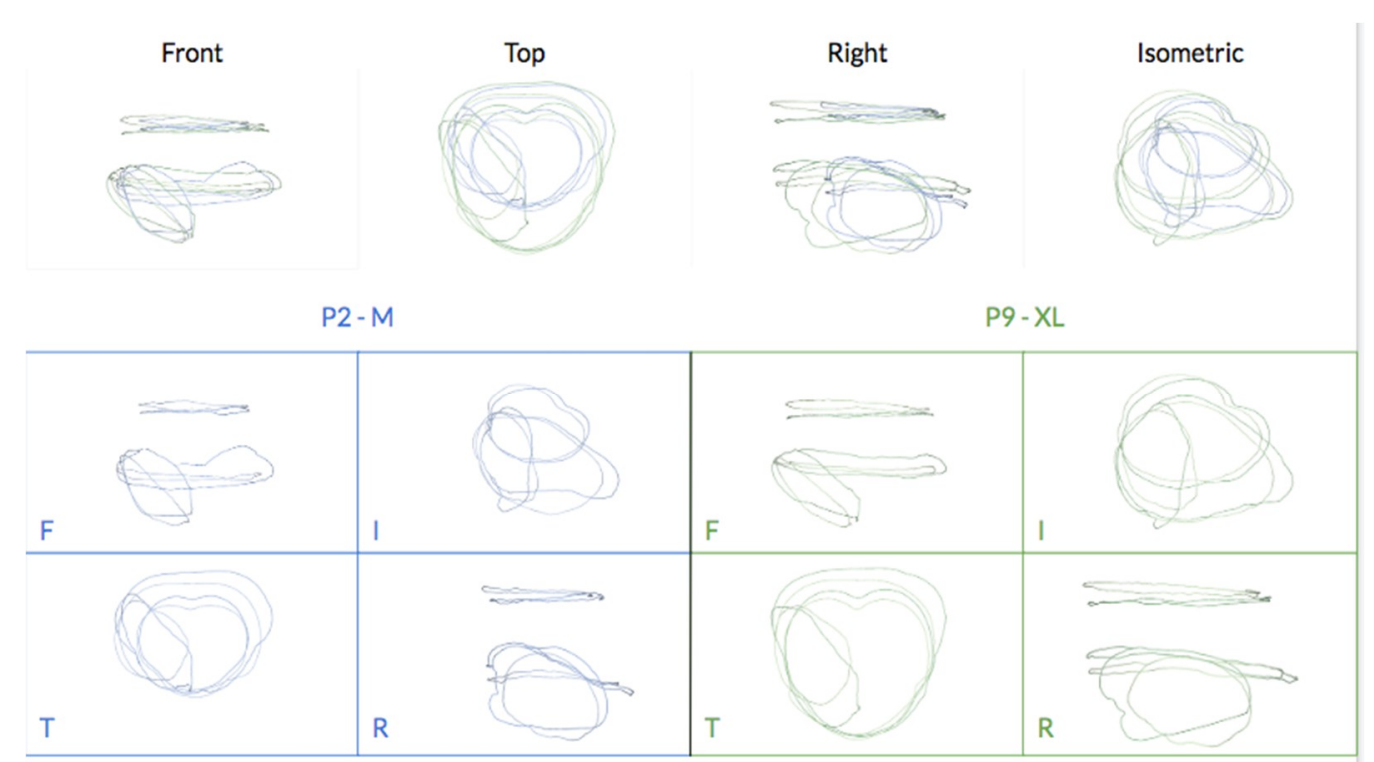

Figure 14. Comparison of waist, hip and pant line curves extracted from a medium and XLarge participant.

\section{Discussion and Conclusion}

\subsection{Overview}

The purpose of this research was to test a new method of body scanning and landmarking the body to enable new ways of analyzing 1D, 2D, and 3D measurements in the seated and standing postures. Movement and shape change of the body occurs on a daily basis and must be examined with respect to product design. This anthropometric study to understand the dynamic position of the body offers a new perspective to improve the design of products worn close to the body. New study methodologies to measure the waist-hip-thigh region must adapt to meet the capabilities of new scanning technology.

While some previous landmarking methods, such as stamping, incremental spacing, and all-over speckle pattern, can quantify skin deformation, previous methods tend not to be able to track the specific dimension change of critical anthropometric measurements used in apparel pattern-making. This landmarking method enables researchers to track circumference measurement and shape change in standard areas, as well as track length and product features.

Traditional anthropometric measures fail to quantify regional shape change that should be incorporated into garment design. Incorporating complex measurements into the development of wearable products ensures better fit and function of garments worn close to the body.

\subsection{Limitations and Future Research}

One limitation to our analysis was the limited functionality of current anthropometric software in the 2D and 3D space. Traditional anthropometric software is designed to capture 1D measurements or 2D flat surfaces. On the body, horizontal and vertical 2D planes in the standing position become 3D curved surfaces in the seated position. Traditional anthropometric measurement software and many 3D analysis software do not have the capability of comparing or visualizing a 3D curved surface to a $2 \mathrm{D}$ plane. Therefore, these limited measurement techniques fail to quantify the 3D complexity of the human body. More evaluation and research into different software is needed to provide a holistic assessment of the body in dynamic postures for 3D scanning.

Future research includes expanding the method to 100 female participants, age 40-65. The researchers will also apply the integrated analysis including 1D, 2D and 3D measurements of the human body to the design of close-to-skin products to improve fit and performance for dynamic movement.

\subsection{Implications and Conclusion}

When considering the implications of this type of data on product, it is important to consider why fit is so integral to the function and comfort of wearable products. Bad fit in one area of the body can cause a domino of bad fit in near-by areas. If a circumference of the garment does not accommodate body movement, it can cause functional features to shift or the circumference itself to shift. Additionally, if a product does not accommodate dimensional change, the worn product will shift towards the smallest 
circumference in the body. This product shift can cause: the garment to 'ride-up' and bunch, discomfort such as pinching and rubbing, the loss of functionality, and can decrease the longevity of the product by weakening the material, ripping, and tearing. As designers and engineers, if we want to improve the product, we must first understand the body.

Examining simple circumference change in order to understand body movement does not reveal the whole story. Areas of the body are expanding and lengthening, contracting and shortening, at different rates. In order to improve the fit of function of products, it's critical to understand how measurements are influencing one another, and how volume and shape change. Product lines of a garment typically cross several circumferences (such as the pantline of underwear), and how each circumference and area of the body changes during movement impacts the functionality and fit of the product.

Products need to integrate stretch to accurately reflect the body's expansion and contraction. This data can improve the development of materials, trims, pattern design, and sizing systems. New 3D scanning methods to quantify diverse bodies can improve a company's competitive advantage through enhanced product fit and inclusive, quality design for all.

\section{References}

[1] S. Choi and S. P. Ashdown, "3D body scan analysis of dimensional change in lower body measurements for active body positions," Textile Research Journal, vol. 81, no. 1, pp. 81-93, 2011.

[2] J. Choi and K. Hong, "3D skin length deformation of lower body during knee joint flexion for the practical application of functional sportswear," Applied Ergonomics, vol. 48, pp. 186-201, 2015.

[3] K. M. Robinette, S. Blackwell, H. Daanen, M. Boehmer, and S. Fleming, "Civilian American and European Surface Anthropometry Resource (CAESAR), Final Report. Volume 1. Summary," SYTRONICS INC DAYTON OH, 2002.

[4] "Size USA." [Online]. Available: http://www.sizeusa.com/. [Accessed: 18-Sep-2019].

[5] "Size North America - Welcome." [Online]. Available: http://www.sizenorthamerica.com/cms/ front content.php?idcat=54\&lang=1. [Accessed: 18-Sep-2019].

[6] T. Kuebler, A. Luebke, J. Campbell, and T. Guenzel, "Size North America-The New North American Anthropometric Survey," in International Conference on Human-Computer Interaction, 2019, pp. 88-98.

[7] L. Griffin, N. Kim, R. Carufel, S. Sokolowski, H. Lee, and E. Seifert, "Dimensions of the Dynamic Hand: Implications for Glove Design, Fit, and Sizing," in International Conference on Applied Human Factors and Ergonomics, 2018, pp. 38-48.

[8] A. M. Wessendorf and D. J. Newman, "Dynamic understanding of human-skin movement and strain-field analysis," IEEE Transactions on Biomedical Engineering, vol. 59, no. 12, pp. 3432-3438, 2012.

[9] R. Granberry, "Active-Contracting Fabrics for Wearable Compression Applications," 2018.

[10]I. ISO, "7250: Basic human body measurements for technological design," International Organization for Standardization, 2017.

[11]E. W. Obropta and D. J. Newman, "Skin strain fields at the shoulder joint for mechanical counter pressure space suit development," in 2016 IEEE Aerospace Conference, 2016, pp. 1-9.

[12] Z. Chen, X. Shao, X. He, J. Wu, X. Xu, and J. Zhang, "Noninvasive, three-dimensional full-field body sensor for surface deformation monitoring of human body in vivo," Journal of biomedical optics, vol. 22, no. 9, p. 095001, 2017.

[13] L. Griffin, S. Sokolowski, and E. Seifert, "Process Considerations in 3D Hand Anthropometric Data Collection: 3DBODY. TECH 2018-9th International Conference and Exhibition on 3D Body Scanning and Processing Technologies, Lugano, Switzerland, 16-17 Oct. 2018," 2018.

[14]K. Langer, "Zur Anatomie und Physilogie der Haut," Sitzungsbericht Der MathematischNaturwissenschaftlichen Classe Der Kaiserlichen Academie Der Wissenschaften, vol. 44, pp. 1948, 1861.

[15]A. S. Iberall, "The use of lines of nonextension to improve mobility in full-pressure suits," RAND DEVELOPMENT CORP CLEVELAND OH, 1964.

[16]S. Luo, J. Wang, X. Yao, and L. Zhang, "A novel method for determining skin deformation of lower limb in cycling," The Journal of The Textile Institute, vol. 108, no. 9, pp. 1600-1608, 2017.

[17]Z. Hu, L. Liu, Z. Liu, and M. Xing, "Dynamic Understanding of Human-Skin Movement and Garment Design of Golf Apparel," in International Conference on Applied Human Factors and Ergonomics, 2017, pp. 982-994.

[18] J Williams, "4D Scanner | Perceiving Systems - Max Planck Institute for Intelligent Systems." [Online]. Available: https://is.tuebingen.mpg.de/. [Accessed: 18-Sep-2019] 